\title{
Inhibition of DNA-PKcs enhances radiosensitivity and increases the levels of ATM and ATR in NSCLC cells exposed to carbon ion irradiation
}

\author{
LINA YANG ${ }^{1-3}$, YUANYUAN LIU $^{2}$, CHAO SUN $^{2}$, XINRUI YANG ${ }^{1,2}$, ZHEN YANG $^{1,2}$, JUNTAO RAN $^{3}$, \\ QIUNING ZHANG ${ }^{3}$, HONG ZHANG $^{2}$, XINYU WANG ${ }^{1}$ and XIAOHU WANG ${ }^{1-3}$ \\ ${ }^{1}$ Institute of Cell Biology, School of Life Sciences, Lanzhou University; ${ }^{2}$ Department of Heavy Ion Radiation Medicine, \\ Institute of Modern Physics, Chinese Academy of Sciences, Lanzhou, Gansu 730000; ${ }^{3}$ Department of Radiotherapy, \\ Gansu Province Tumor Hospital, Lanzhou, Gansu 730005, P.R. China
}

Received April 3, 2015; Accepted August 20, 2015

DOI: 10.3892/ol.2015.3730

\begin{abstract}
Non-small cell lung cancer (NSCLC) exhibits radioresistance to conventional rays, due to its DNA damage repair systems. NSCLC may potentially be sensitized to radiation treatment by reducing those factors that continuously enhance the repair of damaged DNA. In the present study, normal lung fibroblast MRC-5 and lung cancer A549 cells were treated with NU7026 and CGK733, which are inhibitors of the DNA-dependent protein kinase catalytic subunit (PKcs) and ataxia telangiectasia mutated (ATM) and ataxia telangiectasia and Rad3-related (ATR), respectively, followed by exposure to X-rays and carbon ion irradiation. The cytotoxic activity, cell survival rate, DNA damage repair ability, cell cycle arrest and apoptosis rate of the treated cells were analyzed with MTT assay, colony formation assay, immunofluorescence and flow cytometry, respectively. The transcription and translation levels of the ATM, ATR and DNA-PKcs genes were detected by reverse transcription-quantitative polymerase chain reaction and western blotting, respectively. The results indicated that the radiosensitivity and DNA repair ability of A549 cells were reduced, and the percentages of apoptotic cells and those arrested at the $\mathrm{G}_{2} / \mathrm{M}$ phase of the cell cycle were significantly increased, following ionizing radiation with inhibitor-pretreatment. The expression levels of ATM, ATR, DNA-PKcs and phosphorylated histone H2AX, a biomarker for DNA double-strand breaks, were all upregulated at the
\end{abstract}

Correspondence to: Professor Xinyu Wang,Institute of Cell Biology, School of Life Sciences, Lanzhou University, 222 Tianshui South Road, Chengguan, Lanzhou, Gansu 730000, P.R. China

E-mail: wangxy@lzu.edu.cn

Professor Xiaohu Wang, Department of Radiotherapy, Gansu Province Tumor Hospital, 2 Xiaoxihu East Road, Lanzhou, Gansu 730005, P.R. China

E-mail: xhwanggansu@163.com

Key words: ATM, ATR, carbon ion, DNA-PKcs, radiosensitivity transcriptional or translational level in A549 cells treated with carbon ion irradiation, compared with the control and X-rays-treated cells. In addition, the treatment with 5-50 $\mu \mathrm{M}$ NU7026 or CGK733 did not produce any obvious cytotoxicity in MRC-5 cells, and the effect of the DNA-PKcs-inhibitor on enhancing the radiosensitivity of A549 cells was stronger than that observed for the ATM and ATR-inhibitor. These findings demonstrated a minor role for ATM and ATR in radiation-induced cell death, since the upregulation of ATM and ATR did not rescue the A549 cells subjected to ionizing irradiation. Therefore, future studies on DNA-PKcs, ATM and ATR may lead to novel specific therapies that supplement general radiotherapy for the treatment of lung cancer.

\section{Introduction}

Lung cancer is responsible for the majority of mortalities from cancer worldwide, and is classified into small-cell lung cancer (SCLC) or non-small cell lung cancer (NSCLC) (1-3). NSCLC is observed in $\leq 85 \%$ of cases of lung cancer, and its overall 5-year survival rate is $17.1 \%$ (1-3). The radiotherapy treatment for NSCLC via conventional low-linear energy transfer (LET) $\mathrm{X}$-rays and $\gamma$-rays (typically used to treat malignant cancer) presents disadvantages, including radiation-induced lung injury and radioresistance of lung cancer cells $(4,5)$. By contrast, high-LET carbon ions possess several advantages on cancer treatment, including higher relative biological effectiveness, lower oxygen enhancement ratio and more uniform depth dose distribution than low-LET rays (6). However, the detailed molecular mechanisms underlying heavy ion radiotherapy and its ability to reduce cellular radioresistance remain largely unclear.

Intrinsic radioresistance, cell proliferation and hypoxia are the 3 major radioresistance mechanisms in cancer cells $(7,8)$. The problems associated with cell proliferation and hypoxia may be eliminated by accelerated radiotherapy with hypoxia modification. However, intrinsic radioresistance is a complex problem difficult to eliminate, due to the multiple signaling pathways involved $(7,8)$. Previous studies have demonstrated that the phosphoinositide-3 kinase (PI3K)/protein kinase B (Akt) 
and $\mathrm{PI} 3 \mathrm{~K} / \mathrm{mammalian}$ target of rapamycin (mTOR) signaling pathways may be frequently activated in certain cancer cells, regulating cellular processes such as proliferation, apoptosis and survival (8-10). The PI3K family includes DNA-dependent protein kinase catalytic subunit (PKcs), ataxia telangiectasia mutated (ATM) and ataxia telangiectasia and Rad3-related (ATR), which participate in the DNA damage response pathway (11). DNA-PKcs and ATM are involved in non-homologous end joining (NHEJ) and homologous recombination (HR) repair of DNA double-strand breaks (DSBs) (12), while ATR is involved in the DNA damage response pathway to repair interrupted replication forks and extensive lesions in single-strand breaks (SSBs) (13). Previous studies on the signaling differences between the DNA damage response to $\gamma$-rays and carbon ion irradiation observed an increased number of ATM and ATR foci following carbon ion irradiation, and suggested that the differences in DNA damage response to low- or high-LET may be due to distinct macromolecular complexes (14). Similarly, Okayasu et al (15) noticed that radiation with iron ions at 2 Gy dose induced complex DNA damage, which was not repaired by the NHEJ pathway. Since members of the PI3K family participate in maintaining the genomic integrity and chromosome stability, it has been hypothesized that these physiological processes may be associated with the radiosensitivity of NSCLC cells.

In the present study, the DNA-PKcs-inhibitor NU7026 and the ATM and ATR-inhibitor CGK733 were used to disrupt the NHEJ repair pathway, in order to investigate the potential alterations in the transcription and translation levels of the ATM, ATR, DNA-PKcs genes, and to determine the radiosensitivity of lung cancer A549 cells exposed to ionizing radiation. The results suggested that the upregulation of ATR/ATM potentially enhanced cellular radiosensitivity in A549 cells treated with the DNA-PKcs-inhibitor, since part of the DNA damage-sensing apparatus was inhibited following carbon ion irradiation. Therefore, high-LET carbon ions instead of low-LET X-rays may be used in the future to treat patients with lung cancer in the clinic. Further studies are required to investigate the potential use of DNA-PKcs, ATM and ATR in specific gene-radiotherapy approaches for the treatment of lung cancer.

\section{Materials and methods}

Cell culture and irradiation treatment. Normal lung fibroblast MRC-5 and lung cancer A549 cells were purchased from the American Type Culture Collection (Manassas, USA), and cultured in minimum essential medium and Dulbecco's modified eagle medium (Gibco Life Technologies, Carlsbad, USA) supplemented with $10 \%$ fetal bovine serum (HyClone, GE Healthcare Life Sciences, Logan, USA), respectively. The cells were incubated in humidified atmosphere at $37^{\circ} \mathrm{C}$ in the presence of $5 \% \mathrm{CO}_{2}$ to maintain exponential cell growth.

A549 cells were irradiated at room temperature with $6 \mathrm{MV}$ X-rays delivered by a PRIMUS linear accelerator (Siemens AG, Berlin, Germany) located in the Gansu Province Tumor Hospital (Lanzhou, China), at a dose rate of $200 \mathrm{cGy} / \mathrm{min}$ and source skin distance of $100 \mathrm{~cm}$; or with $300 \mathrm{MeV}$ carbon ion $\left({ }^{12} \mathrm{C}^{6+}\right)$ beams, provided at a dose rate of $1 \mathrm{~Gy} / \mathrm{min}$ and LET of $49 \mathrm{KeV} / \mu \mathrm{m}$, at the Heavy Ion Research Facility in Lanzhou (Institute of Modern Physics, Chinese Academy of Sciences, Lanzhou, China). The cells were exposed to $2 \mathrm{~Gy}$, and radiation doses were determined based on previous pilot studies $(11,13,14)$. Non-irradiated A549 cells were handled in parallel with the irradiated cells.

MTT assay. MRC-5 and A549 cells were plated into 96-well dishes at a density of $5 \times 10^{4}$ cells/well. NU7026 and CGK733 (Abcam, Cambridge, UK) were added to each well at a final concentration of 5-50 $\mu \mathrm{M}$, and incubated for $48 \mathrm{~h}$. Thereafter, MTT (final concentration, $5 \mathrm{mg} / \mathrm{ml}$ ) was added to each well. The medium was then removed, and the formazan crystals were dissolved by adding $150 \mu \mathrm{l}$ dimethyl sulfoxide. The absorbance at $490 \mathrm{~nm}$ was subsequently measured in a microplate reader (Infinite M200; Tecan Group Ltd., Männedorf, Switzerland) $(16,17)$.

Colony formation assay. A549 cells (2,000 cells) were seeded in a culture dish of $100 \mu \mathrm{m}$ in diameter, and treated with $10 \mu \mathrm{M}$ NU7026 or CGK733 for $30 \mathrm{~min}$, prior to be exposed to 2 Gy $\mathrm{X}$-ray and carbon ion irradiation. Following the addition of fresh medium, cell incubation continued under standard culture conditions $\left(37^{\circ} \mathrm{C}\right.$ and $\left.5 \% \mathrm{CO}_{2}\right)$. The cells were washed with phosphate-buffered saline (PBS), fixed with ethanol and stained with Giemsa 10 days later. The number of colonies was calculated using a Multi Image ${ }^{\mathrm{TM}}$ Light Cabinet and the AlphaEase ${ }^{\mathrm{TM}}$ software (Alpha Innotech Corporation, San Leandro, CA, USA).

Cell cycle analysis. Cells were harvested and fixed in $70 \%$ ice-cold ethanol for $>48 \mathrm{~h}$ at $-20^{\circ} \mathrm{C}$. Subsequently, the cells were washed twice with PBS, resuspended in propidium iodide (PI) staining solution $(5 \mu \mathrm{g} / \mathrm{ml} \mathrm{PI}, 10 \mathrm{kU} / \mathrm{ml}$ RNaseA and $0.005 \%$ Triton X-100 in PBS) at a concentration of $1 \times 10^{6}$ cells $/ \mathrm{ml}$, and incubated in the dark at room temperature for $30 \mathrm{~min}$. The cell cycle distribution was analyzed using a FACScan flow cytometer (BD Biosciences, Franklin Lakes, USA) and FlowJo version 7.6 software (FlowJo, LLC, Ashland, USA).

$\gamma H 2 A X$ foci immunofluorescence. Cells were seeded in a 6 -well plate at a density of $1 \times 10^{5}$ cells/well, and covered with a coverslip for $24 \mathrm{~h}$ to allow the cells to attach. Next, the cells were treated with NU7026 for $30 \mathrm{~min}$, prior to being subjected to $2 \mathrm{~Gy}$ X-rays and carbon ion irradiation. At $0.5 \mathrm{~h}$ and $24 \mathrm{~h}$ post-irradiation, the cells were fixed with $4 \%$ paraformaldehyde for $15 \mathrm{~min}$, and then treated with $0.1 \%$ Triton X-100 for $30 \mathrm{~min}$ and $5 \%$ bovine serum albumin (BSA) for $1 \mathrm{~h}$. Next, the cells were incubated overnight at $4^{\circ} \mathrm{C}$ with primary monoclonal antibody anti- $\gamma$ H2AX (1:500; Bioworld Technology, Inc., St. Louis, MO, USA) in the presence of $1 \%$ BSA. Cells were washed 3 times with PBST $(0.05 \%$ Tween 20$)$ for $10 \mathrm{~min}$, and incubated at room temperature for $1 \mathrm{~h}$ with IgG-fluorescein isothiocyanate in the presence of 1\% BSA (1:5000; Cell Signaling Technology, Inc.). Following the addition of $1.5 \mu \mathrm{g} / \mathrm{ml}$ 4',6-diamidino-2-phenylindole (DAPI) to counterstain the nuclei. The coverslip containing the cells was then mounted with VECTASHIELD® Antifade Mounting Medium (Vector Laboratories, Inc., Burlingame, CA, USA). $\gamma \mathrm{H} 2 \mathrm{AX}$ foci were detected with a LSM 700 laser scanning confocal microscope (Zeiss, Oberkochen, Germany).

Reverse transcription-quantitative polymerase chain reaction $(R T-q P C R)$. Total RNA was isolated from A549 cells with TRIzol (Invitrogen Life Technologies, Carlsbad, USA). 
cDNA was synthesized with a PrimeScript ${ }^{\mathrm{TM}} \mathrm{RT}$ reagent kit (Takara Biotechnology Co., Ltd., Dalian, China) in a total volume of $20 \mu \mathrm{l}$. Then, multiplex qPCR was performed in a total volume of $25 \mu$ l, using SYBR Green Master Mix (Takara Biotechnology Co., Ltd.), $50 \mathrm{ng}$ DNA and $10 \mu \mathrm{M}$ of each of the following primers from Takara Biotechnology Co., Ltd.: DNA-PKcs (forward, 5'-AGGGAAGAAGAGTCT CTGGTGG-3'; reverse, 5'-ATTAGGGGATCTGTTGCC TGGC-3') (18); ATM (forward, 5'-ACTATCCCAATACAC TGCTGGAGA-3'; reverse, 5'-TTTGAGCAACTGACTGGC AAAC-3'); ATR (forward, 5'-CCAAAGCGCCACTGA ATGAA-3'; reverse, 5'-ACCTTGTAGTCGCTGCTCAAT GTC-3'); and GAPDH (forward, 5'-GAAGGTGAAGGTCGG AGTC-3'; reverse, 5'-GAAGATGGTGATGGGATTTC-3').

The reaction was conducted on an FTC-3000 qPCR system (Shanghai Funglyn Biotech Co., Ltd., Shanghai, China), with the following cycling conditions: $30 \mathrm{sec}$ at $95^{\circ} \mathrm{C}, 40$ cycles of $5 \mathrm{sec}$ at $95^{\circ} \mathrm{C}$ and $30 \mathrm{sec}$ at $59^{\circ} \mathrm{C}$. Each PCR was repeated 3 times. The relative gene quantification approach $\left(2^{-\Delta \Delta C t}\right)$ was used according to the method previously described (19).

Western blotting. A549 cells were lysed in lysis buffer supplemented with $1 \mathrm{mM}$ phenylmethylsulfonyl fluoride, and the protein concentration in the cell lysate was determined using a BCA assay kit (Pierce Biotechnology Inc., Rockford, USA). $20 \mu \mathrm{g}$ of proteins from the whole-cell lysate was mixed with SDS buffer, separated on SDS-PAGE at $80 \mathrm{~V}$ for $2 \mathrm{~h}$ and transferred to poly vinylidene difluoride membranes (Bio-Rad Laboratories, Inc.) for $2 \mathrm{~h}$. The membranes were then blocked for $1 \mathrm{~h}$ with PBS containing 5\% BSA (Sigma-Aldrich), and incubated with the corresponding primary monoclonal rabbit antibody IgG anti-ATM, ATR, DNA-PKcs or $\beta$-actin (Cell Signaling Technology, Inc., Danvers, MA, USA) $(1: 1,000)$ at $4^{\circ} \mathrm{C}$ overnight. Next, the membranes were washed with PBST for $30 \mathrm{~min}$, and incubated with a goat anti-rabbit horseradish peroxidase-conjugated secondary antibody $(1: 5,000$, Cell Signaling Technology, Inc.) for $1 \mathrm{~h}$ at room temperature. Following 3 washes with PBST for $10 \mathrm{~min}$, a chemiluminescence kit (Santa Cruz Biotechnology, Inc., Dallas, TX, USA) was used to detect the reactive proteins. The data were represented as values relative to the protein levels of $\beta$-actin.

Statistical analysis. The data, presented as the mean \pm standard deviation from $\geq 3$ independent experiments, were evaluated for statistical significance with the Student's t-test, using Microsoft Excel software (Microsoft Corporation, Redmond, USA). $\mathrm{P}<0.05$ was considered to indicate a statistically significant difference.

\section{Results}

The cytotoxicity of NU7026 and CGK733 was evaluated in A549 and MRC-5 cells. The effect of NU7026 or CGK733 on cell proliferation was determined by MTT assay following 48-h exposure of A549 and MRC-5 cells to the above inhibitors. At concentrations of NU7026 and CGK733 $<10 \mu \mathrm{M}$, MRC-5 and A549 cells exhibited 2 and $10 \%$ inhibition rate, respectively, whereas at concentrations of NU7026 and CGK733 $>20 \mu \mathrm{M}$, the inhibition rate of MRC-5 and A549 cells increased to $>8$ and $16 \%$, respectively (Fig. 1).

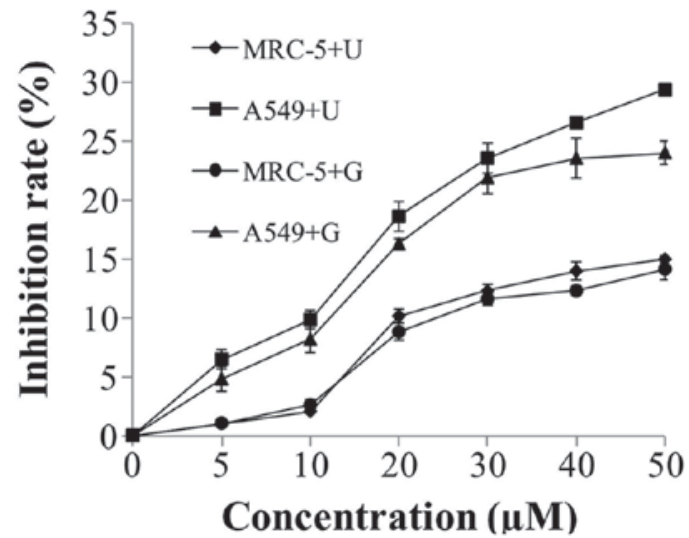

Figure 1. Effect of NU7026 and CGK733 on the proliferation rate of MRC-5 and A549 cells following 48-h exposure. U, NU7026; G, CGK733.

A
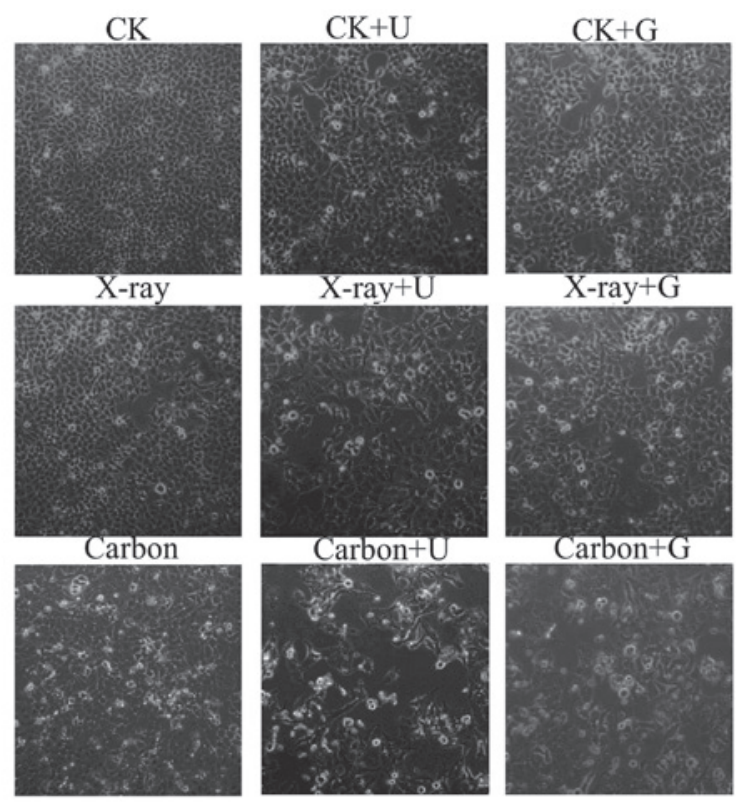

B

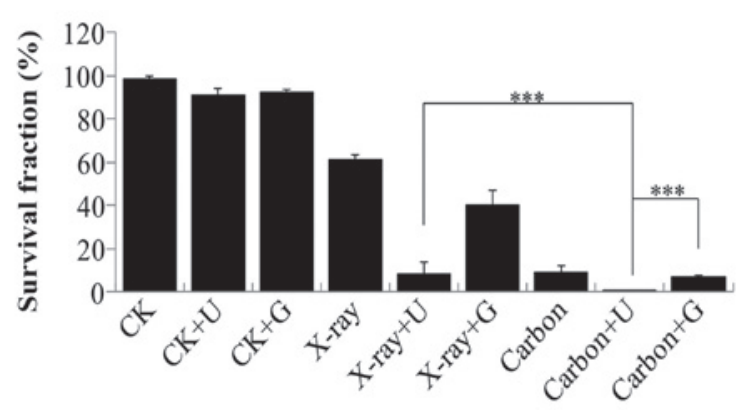

Figure 2. (A) Treatment of A459 cells with NU7026 or CGK733 altered the cell morphology and reduced the number of cells, following irradiation. A459 cells were incubated with $10 \mu \mathrm{M}$ NU7026 or CGK733 for $30 \mathrm{~min}$, prior to irradiation with X-ray or carbon ions (magnification, x100). (B) Following incubation with NU7026 or CGK733 for 10 days, the number of colonies containing $>50$ cells were counted to calculate the surviving fraction. $\mathrm{CK}$, non-irradiated A549 cells; U, NU7026; G, CGK733. ${ }^{* * *} \mathrm{P}<0.001$.

Compared with A549 cells treated with 5-50 $\mu \mathrm{M}$ NU7026 or CGK733, no obvious cytotoxicity was observed in MRC-5 cells, following treatment with NU7026 or CGK733 for 
$0.5 \mathrm{~h}$

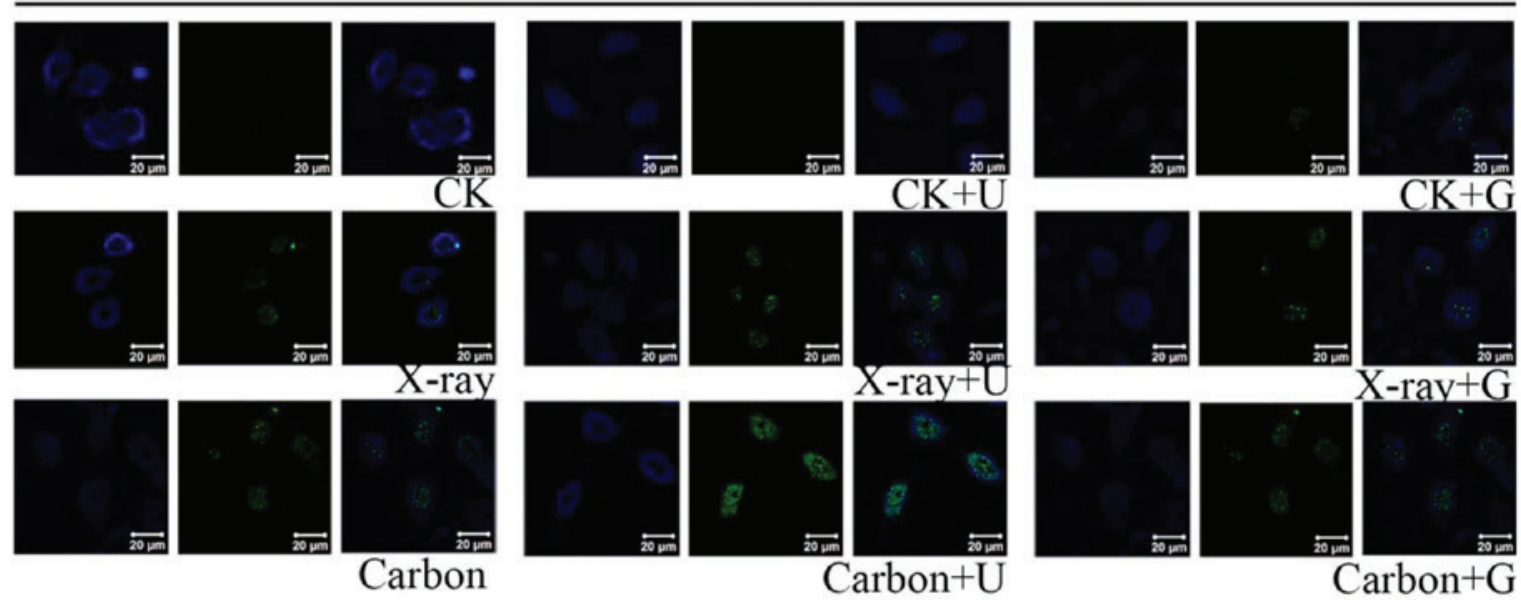

$24 \mathrm{~h}$

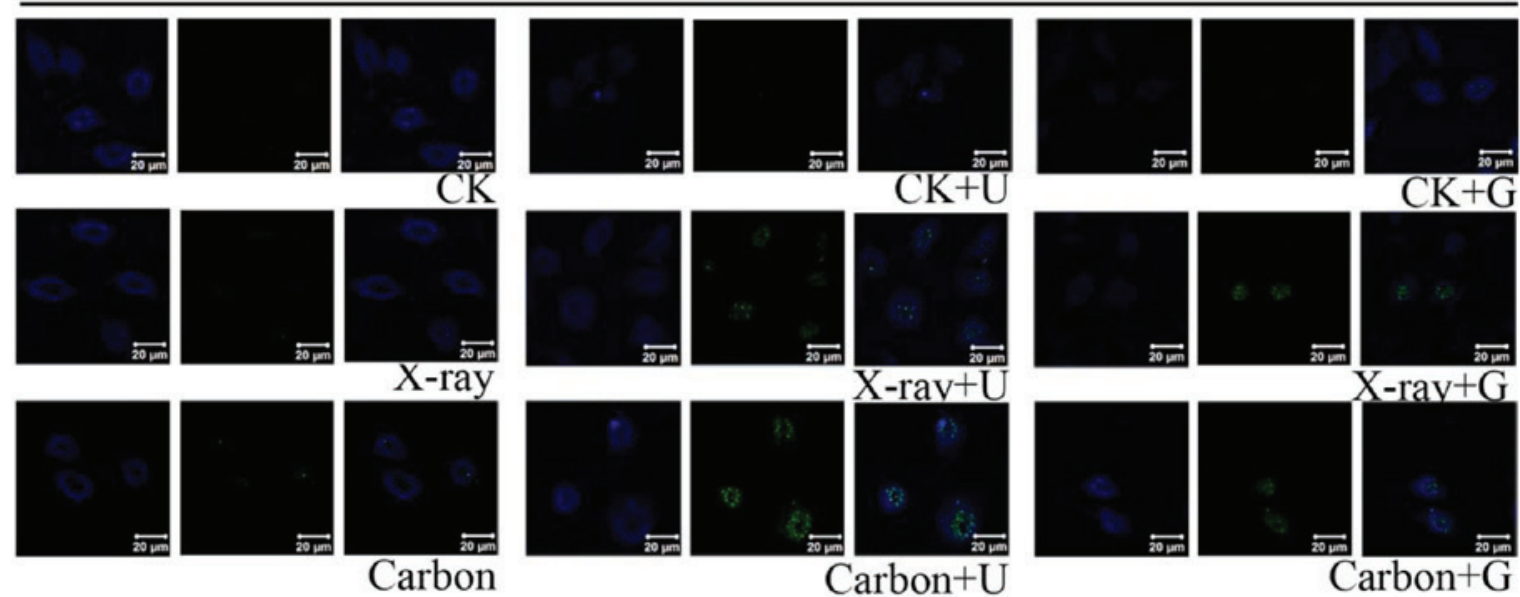

B

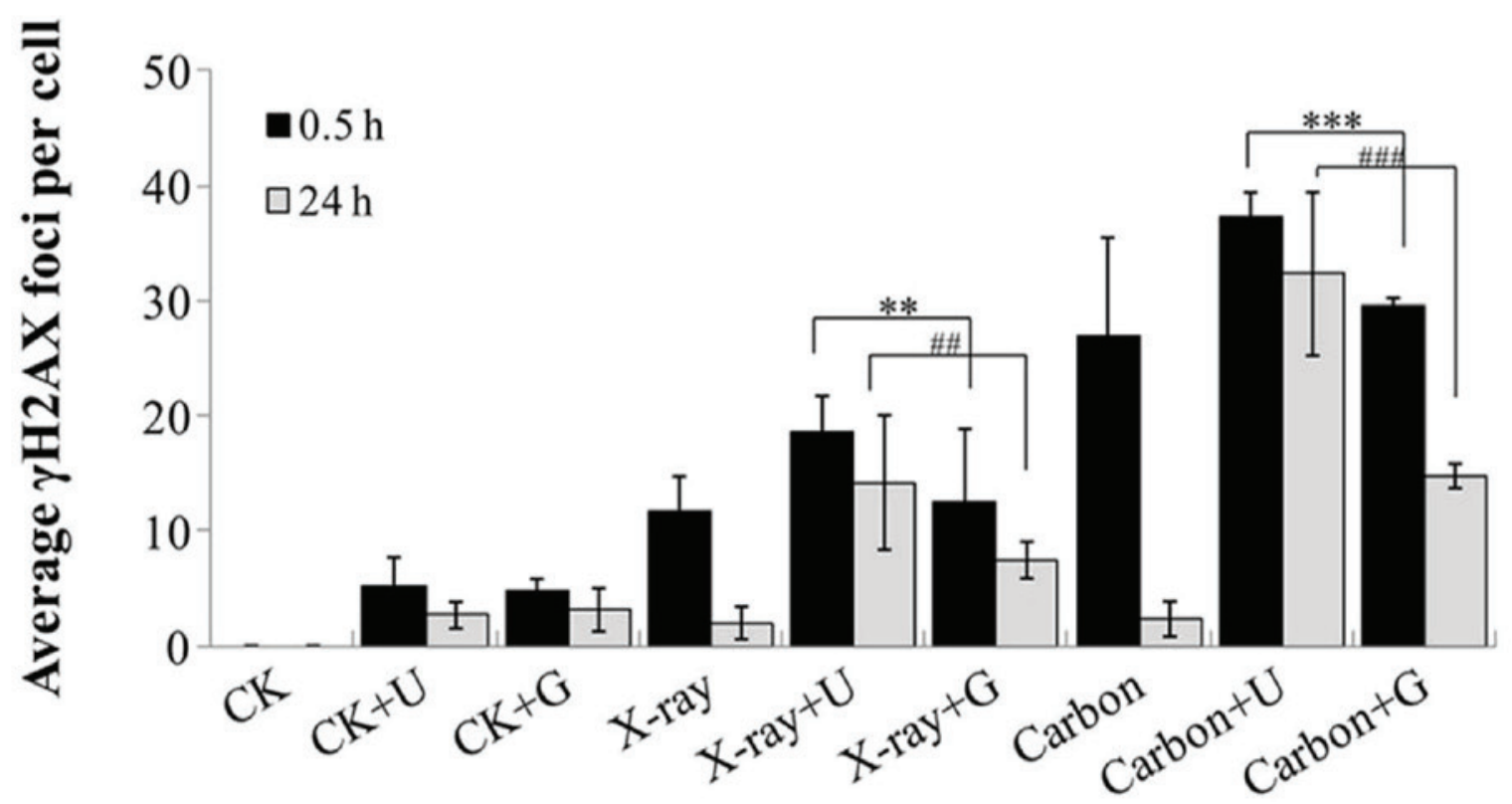

Figure 3. (A) Effect of NU7026 and CGK733 combined with X-rays and carbon ion irradiation on DNA damage and repair in A549 cells. Cell nuclei were stained blue by 4',6-diamidino-2-phenylindole, whereas the $\gamma \mathrm{H} 2 \mathrm{AX}$ foci appear green in the image at 0.5 and $24 \mathrm{~h}$ post-irradiation. The scale bars represent $20 \mu \mathrm{m}$. (B) Statistical analysis of the number of $\gamma \mathrm{H} 2 \mathrm{AX}$ green foci visualized in panel A

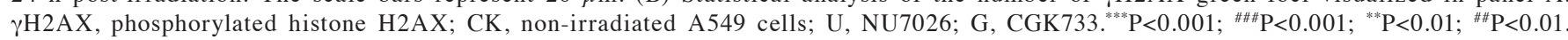


A

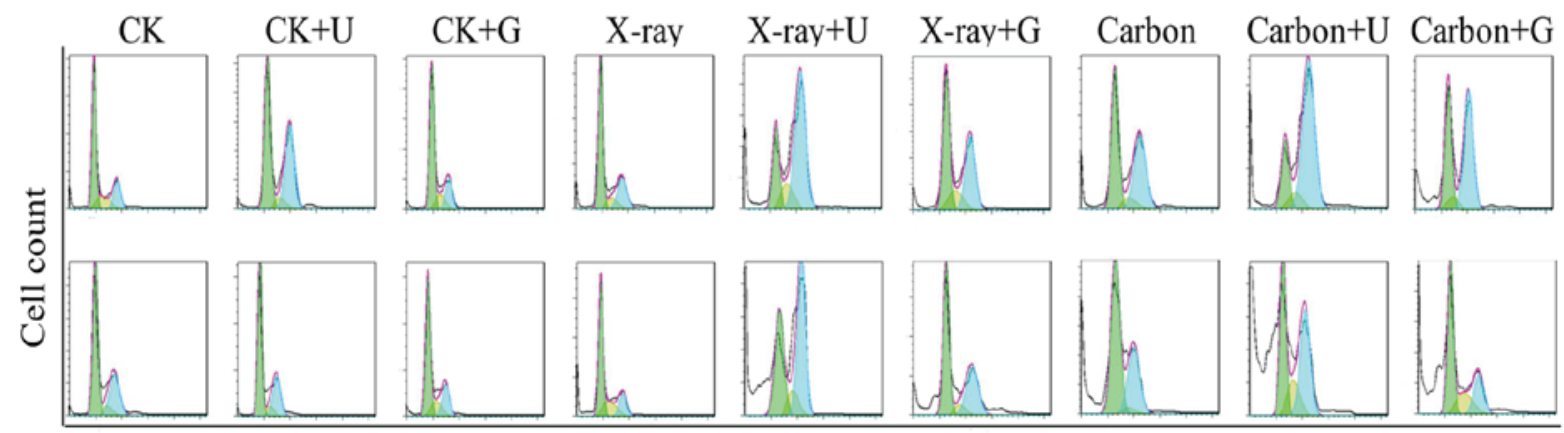

DNA content

B

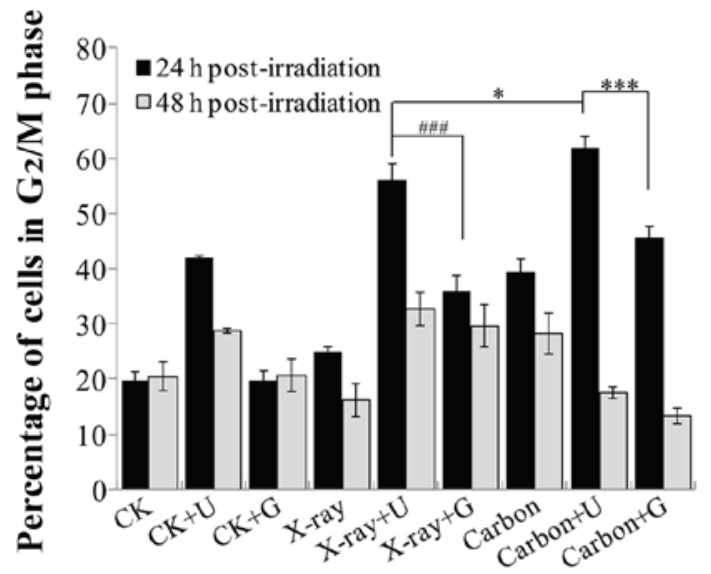

C

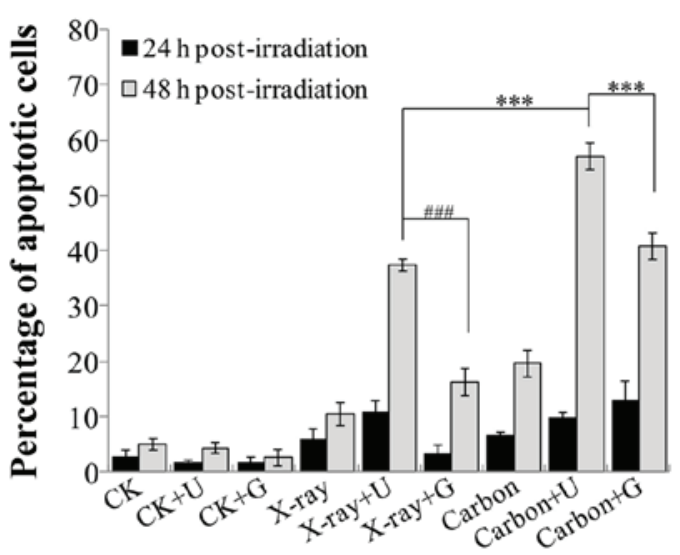

Figure 4. (A) Cell cycle was analyzed in irradiated A456 cells by propidium iodide staining via flow cytometry at 24 and 48 h post-irradiation, following treatment with $10 \mu \mathrm{M}$ NU7026 or CGK733 for $30 \mathrm{~min}$. The percentage of (B) cells arrested at the $\mathrm{G}_{2} / \mathrm{M}$ phase and (C) apoptotic cells was calculated via FlowJo7.6 software from 3 independent experiments. CK, non-irradiated A549 cells; U, NU7026; G, CGK733. ${ }^{* * *} \mathrm{P}<0.001 ;{ }^{\# \# \# P<0.001 ; ~} \mathrm{P}<0.05$.

48 h (Fig. 1). Consequently, $10 \mu \mathrm{M}$ NU7026 and CGK733 were selected to examine the radiosensitivity of A549 cells in subsequent experiments.

NU7026-treatment and irradiation enhance cellular radiosensitivity. A549 cells were pretreated with NU7026 or CGK733 for $30 \mathrm{~min}$, prior to be irradiated. To identify cellular alterations upon inhibitors-treatment and/or irradiation, the cells were analyzed under a microscope. A reduction in the number of cells and alterations in cell morphology were observed (Fig. 2A). Following 10 days, the survival fraction of cells exposed to carbon ion irradiation that had been pretreated with NU7026 reduced to $0.67 \%$, compared with the control group (CK 98.75\%, CK+U 90.82\% and CK+G 92.63\%) and the NU7026-pretreated cells exposed to 2 Gy X-ray irradiation $(8.75 \%)$. The survival fraction of cells exposed to ionizing radiation following CGK733-treatment was higher than that of cells exposed to ionizing radiation following NU7026-treatment (Fig. 2B). These results implied there was a significant increase in the radiosensitivity of A549 cells following NU7026-treatment and carbon ion irradiation.

NU7026-treatment and irradiation reduce DNA damage repair. $\gamma \mathrm{H} 2 \mathrm{AX}$ is a marker of DSBs and also an important factor that recruits downstream proteins in response to
DNA damage (20), $\gamma \mathrm{H} 2 \mathrm{AX}$ foci were detected in A549 cells at $0.5 \mathrm{~h}$ and $24 \mathrm{~h}$ post-irradiation, regardless of prior NU7026 or CGK733-treatment (Fig. 3). The X-ray+NU7026 and carbon+NU7026 groups exhibited a significant increase in $\gamma \mathrm{H} 2 \mathrm{AX}$ foci, compared with the groups treated with irradiation or CGK733 at $0.5 \mathrm{~h}(\mathrm{P}<0.001)$. However, these $\gamma \mathrm{H} 2 \mathrm{AX}$ foci remained unaltered from 0.5 to $24 \mathrm{~h}$ post-irradiation, and did not dephosphorylate to repair DNA damage, indicating that the process of DNA repair had been completed (Fig. 3). Therefore, the X-ray+NU7026 and carbon+NU7026 groups appeared to display a poorer ability to repair DNA damage from 0.5 to $24 \mathrm{~h}$ post-irradiation, compared with the other groups (Fig. 3B). These results indicated that the NU7026-treatment led to a significant reduction in DNA damage repair, compared with the CGK733-treatment, in A459 cells exposed to ionizing radiation.

Exacerbated cell cycle $G_{2} / M$ phase arrest increases cell apoptosis. Cell cycle arrest was examined by flow cytometry, and represented as a blue $\mathrm{G}_{2} / \mathrm{M}$ peak in a DNA histogram (Fig. 4A). In contrast to 2 Gy X-rays irradiation with NU7026-treatment or 2 Gy carbon ion irradiation with CGK733-treatment, a clear increase in the percentage of cells arrested at the $\mathrm{G}_{2} / \mathrm{M}$ phase was observed in NU7026-pretreated A549 cells exposed to 2 Gy carbon ion 


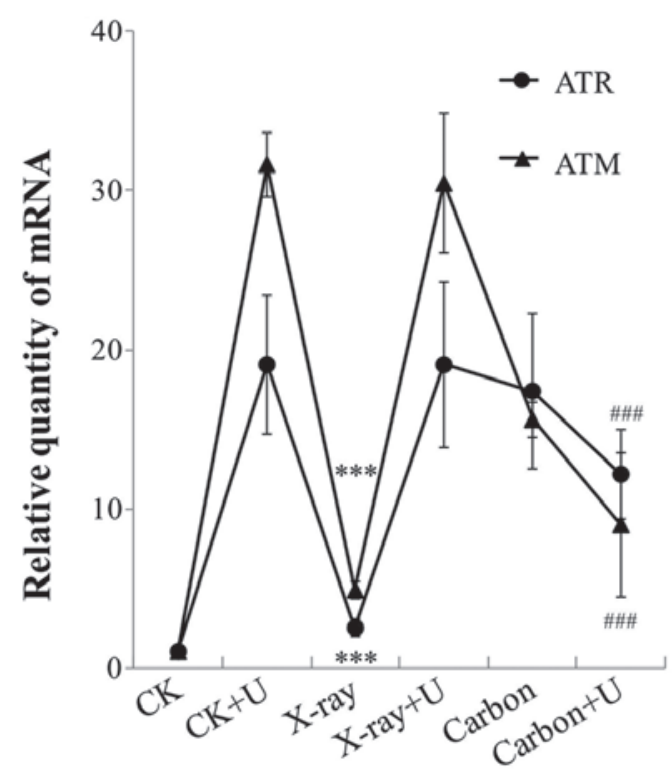

C

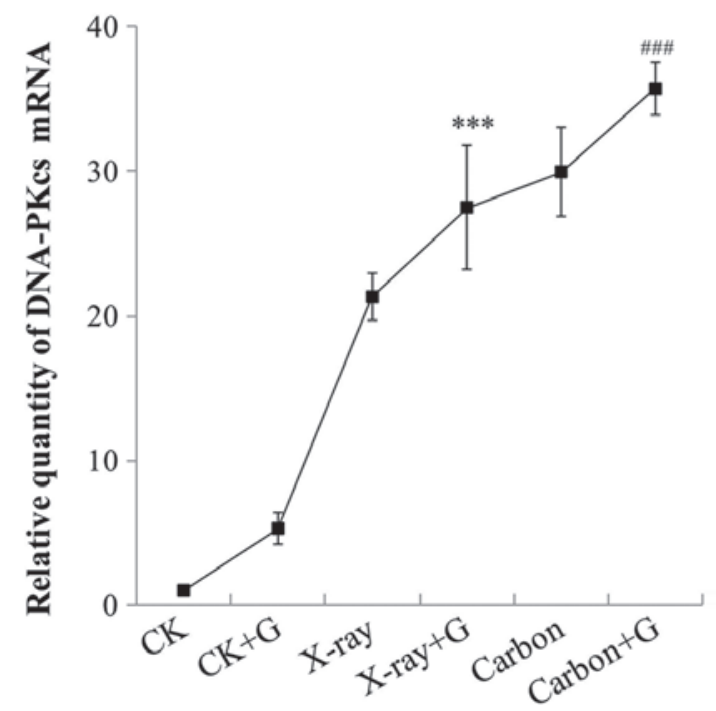

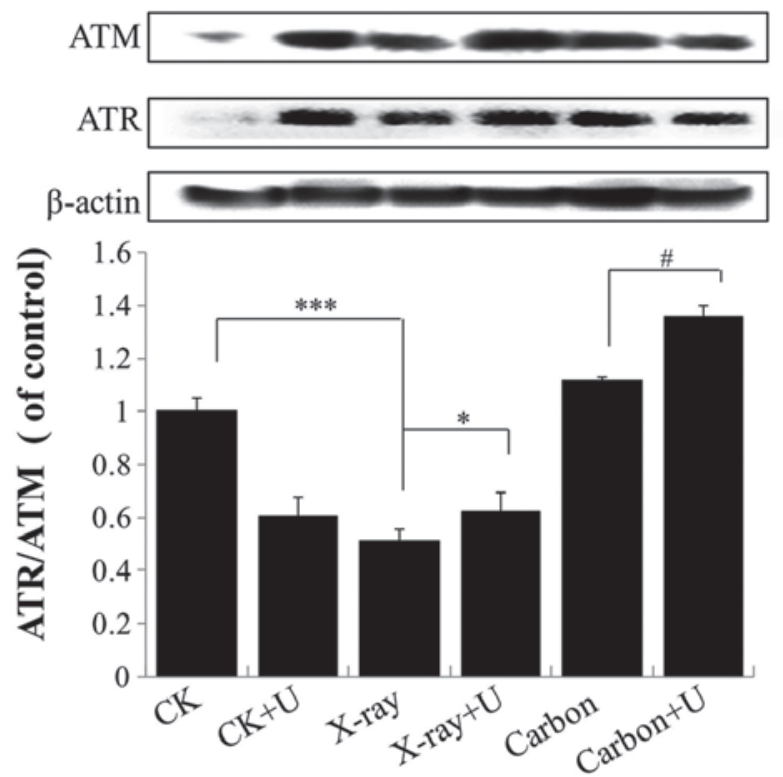

D
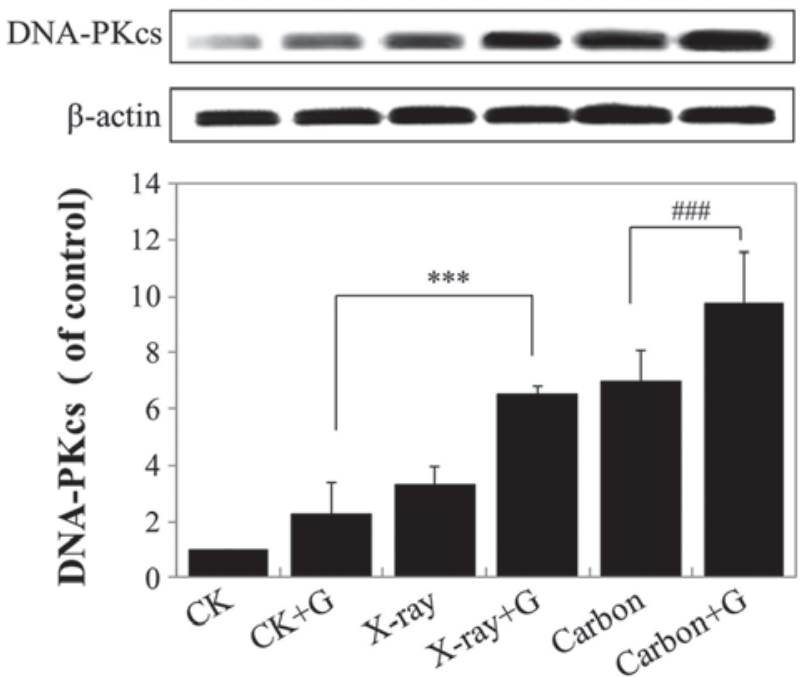

Figure 5. (A) Products derived from the transcription of the ATM and ATR genes were detected by RT-qPCR at 24 h post-irradiation in A459 cells subjected to different LET and/or NU7026-pretreatment. (B) The protein levels of ATM and ATR were determined by western blotting at $24 \mathrm{~h}$ post-irradiation in A549 cells, following exposure to ionizing radiation of different LET and/or NU7026-pretreatment. (C) Products derived from the transcription of DNA-PKcs were detected by RT-qPCR at $24 \mathrm{~h}$ post-irradiation in A459 cells subjected to different LET and/or CGK733-pretreatment. (D) The protein expression levels of DNA-PKcs were detected by western blotting at $24 \mathrm{~h}$ post-irradiation in A459 cells subjected to different LET and/or CGK733-pretreatment. In all cases, A549 cells were incubated with $10 \mu \mathrm{M}$ NU7026 or CGK733 for $30 \mathrm{~min}$ prior to being irradiated. ATM, ataxia telangiectasia mutated; ATR, ataxia telangiectasia and Rad3-related; RT-qPCR, reverse transcription-quantitative polymerase chain reaction; LET, linear energy transfer; DNA-PKcs, DNA-dependent protein kinase catalytic subunit; CK, non-irradiated A549 cells; U, NU7026; G, CGK733 ${ }^{* * *} \mathrm{P}<0.001 ;{ }^{* \# \#} \mathrm{P}<0.001 ;{ }^{*} \mathrm{P}<0.05 ;{ }^{*} \mathrm{P}<0.05$.

irradiation at $24 \mathrm{~h}$ post-irradiation $(\mathrm{P}<0.05$ and $\mathrm{P}<0.001$; Fig. 4B). However, at $48 \mathrm{~h}$ post-irradiation, the number of A549 cells arrested at the $\mathrm{G}_{2} / \mathrm{M}$ phase significantly reduced following $2 \mathrm{~Gy}$ carbon ion irradiation, regardless of the pretreatment with NU7026 or CGK733. Therefore, as the time post-irradiation increased, the fraction of cells in the $\mathrm{G}_{2} / \mathrm{M}$ phase reduced. In contrast, the number of apoptotic cells rapidly increased (Fig. 4C), and the percentage of cells treated with NU7026 or CGK733 at 48 h post-irradiation was higher than at $24 \mathrm{~h}$ post-irradiation. These results indicated that a pronounced $\mathrm{G}_{2} / \mathrm{M}$ arrest may contribute to cell apoptosis.

DNA-PKcs-inhibition enhances the transcription and translation of ATM and ATR. When DNA-PKcs is inhibited, ATM and ATR regulate cell cycle arrest and apoptosis (21). Therefore, RT-qPCR was performed at $24 \mathrm{~h}$ post-irradiation to quantify the relative expression levels of ATM and ATR in A549 cells that had been exposed to 2 Gy irradiation. Compared with 
the CK group (non-irradiated A549 cells), the gene levels of ATM and ATR were markedly upregulated in A459 cells following irradiation, and appeared to decline in A549 cells exposed to carbon ion irradiation plus NU7026-treatment versus NU7026-treatment alone ( $\mathrm{P}<0.001$; Fig. 5A). In addition, the gene expression levels of ATM and ATR gradually increased in A549 cells, following X-rays and carbon ion irradiation alone, compared with the gradual reduction observed in NU7026-treated cells (Fig. 5A). The ability of carbon ion irradiation to regulate the intracellular levels of ATM and ATR was opposite to the effects observed with X-rays irradiation. The results of western blotting for ATM and ATR were in agreement with those from RT-qPCR, and indicated high expression levels of ATR and ATM in A459 cells following carbon ion irradiation alone and carbon irradiation with NU7026-pretreatment, compared with control cells (Fig. 5B).

To investigate the mechanisms that led to the observed reduced survival rate, increased percentage of cells in the $\mathrm{G}_{2} / \mathrm{M}$ phase, and increased apoptosis rate, following X-rays and carbon ion irradiation with or without NU7026-treatment in A459 cells, the expression levels of DNA-PKcs were examined in A549 cells treated with the ATM and ATR-inhibitor CGK733 and ionizing radiation. The gene and protein expression levels of DNA-PKcs were observed to be upregulated in A549 cells exposed to different LET rays and CGK733-pretreatment (Fig. 5C and D). In particular, the pretreatment with CGK733 and carbon ion irradiation significantly enhanced the levels of DNA-PKcs in A549 cells, compared with the other groups $(\mathrm{P}<0.001)$. These findings indicated that the inhibition of DNA-PKcs regulated cell apoptosis and cell cycle arrest following carbon ion irradiation through a mechanism upstream of ATM and ATR.

\section{Discussion}

Previous studies have demonstrated that high-LET radiation may produce high-clustered DNA damage, including DSBs, SSBs, oxidized bases, and apurinic and apyrmidinic sites (22). Thus, the complexity of DNA damage produced by high-LET radiation is higher than that from low-LET radiation (22). DNA-PKcs and ATM exhibit a critical sensory role in DSB repair through the NHEJ pathway in mammalian cells, while ATR is activated by SSBs to alleviate cellular genotoxic stress $(21,23-25)$. DSBs can activate checkpoint signaling, and are usually repaired through the NHEJ and HR pathways (26). HR occurs in the $S$ and $G_{2}$ phases of the cell cycle, whereas NHEJ exists during the whole cell cycle (26). Therefore, the additional inhibitory effect on NHEJ may retard the repair of carbon ion irradiation-induced DNA damage. In the present study, the inhibition of the NHEJ pathway led to the repair of carbon ion-induced DNA damage to become dependent on the slow HR DNA repair pathway. H2AX can be rapidly phosphorylated at Ser139, which is dependent on effector signals upstream of ATM, ATR and DNA-PKcs $(13,27,28)$. Previous studies have indicated that $\gamma \mathrm{H} 2 \mathrm{AX}$ foci may be a sensitive indicator of DSB formation and repair (29). The results of the present study revealed that $\gamma \mathrm{H} 2 \mathrm{AX}$ foci of A549 cells were also increased by mechanisms upstream of ATM and ATR when the cells were treated with NU7026 or CGK733 and radiation for $0.5 \mathrm{~h}$. The number of green foci observed in the irradiated A459 cells markedly reduced with time post-irradiation when the cells were subjected to X-rays and carbon ion irradiation alone, but not following X-rays and carbon ion irradiation with NU7026 or CGK733-pretreatment (Fig. 3). These results indicated that the A459 cells that had been subjected to X-rays and carbon ion irradiation alone were experiencing DNA damage repair, as indicated by the marked reduction in the number of green foci, whereas the DNA damage repair was limited in those cells subjected to X-rays and carbon ion irradiation with NU7026-pretreatment, as indicated by their persistent green foci. The DNA damage repair induced by NU7026-treatment was limited, compared with the CGK733-treatment plus radiation, suggesting that the DNA damage repair ability of DNA-PKcs was stronger than that of ATM and ATR. In addition, a larger number of $\gamma \mathrm{H} 2 \mathrm{AX}$ foci were induced by high-LET particles, compared with low-LET X-rays. However, the downstream repair factors recruited by the formation of $\gamma \mathrm{H} 2 \mathrm{AX}$ foci did not function, leading to the continuous localization of $\gamma \mathrm{H} 2 \mathrm{AX}$ at unrepaired damage sites. These data also suggested that numerous damaged cells arrested in the $\mathrm{G}_{2} / \mathrm{M}$ phase were not capable of being repaired via the slow HR repair pathway alone. Previous studies have demonstrated that the formation of $\gamma \mathrm{H} 2 \mathrm{AX}$ foci depends on the presence of ATM, ATR and DNA-PKcs, following exposure to ionizing radiation $(30,31)$. Furthermore, $\gamma \mathrm{H} 2 \mathrm{AX}$ triggered the $\mathrm{G}_{2} / \mathrm{M}$ checkpoint arrest by mediating the concentration of $\mathrm{p} 53$ binding protein 1 at the DSBs induced by ionizing radiation. Otherwise, the sole presence of these DSBs would be insufficient to prevent the damaged cells from entering mitosis (32).

Previous studies have demonstrated that the principal biological task of the cell cycle is to create time for DNA damage repair. If the repair is successful, the cell cycle continues operating. If the DNA damage is too serious to be repaired, cells will undergo apoptosis $(33,34)$. In the present study, the number of A549 cells arrested at the $\mathrm{G}_{2} / \mathrm{M}$ phase increased at $24 \mathrm{~h}$ post-irradiation when the cells were pretreated with NU7026 or CGK733 (Fig. 4). Previous studies have suggested that cells accumulated in the $\mathrm{G}_{2} / \mathrm{M}$ phase were potentially subjected to apoptosis and sensitive to ionizing radiation $(35,36)$. In the present study, the number of cells arrested at the $\mathrm{G}_{2} / \mathrm{M}$ phase markedly reduced with time post-irradiation, while the number of apoptotic cells increased from 24 to $48 \mathrm{~h}$ post-irradiation. This increase in $\mathrm{G}_{2} / \mathrm{M}$ phase arrest and apoptosis was induced by high-LET particles, compared with low-LET X-rays radiation. Furthermore, the effects exhibited by the DNA-PKcs-inhibitor NU7026 were more pronounced than those displayed by the ATM and ATR-inhibitor CGK733. Therefore, the increase in $\mathrm{G}_{2} / \mathrm{M}$ arrest appeared to enhance the radiosensitivity of A459 cells to carbon ion irradiation via apoptosis-associated cell death.

Previous studies have demonstrated that effectors located upstream of ATM, ATR and DNA-PKcs were able to initiate the $\mathrm{G}_{2} / \mathrm{M}$ cell cycle arrest and DNA damage repair pathway through other kinases $(21,37,38)$. In the present study, upregulation of ATM, ATR and DNA-PKcs in A459 cells was also evident following carbon ion irradiation with NU7026 or CGK733-pretreatment, which indicated that ATR, ATM and DNA-PKcs were involved in DNA damage repair, possibly through regulation of cell 
cycle arrest and apoptosis, rather than through the slow HR pathway. The upregulated ATR/ATM rate in ionized A459 cells observed in the present study was consistent with the results of previous studies $(13,14)$. In addition, the effect of the DNA-PKcs-inhibitor on enhancing the radiosensitivity of A549 cells was stronger than the effect of the ATM and ATR-inhibitor, since the potent repair ability of DNA-PKcs was inhibited following carbon ion irradiation.

In conclusion, in the present study, the treatment with NU7026 increased the cellular radiosensitivity of lung cancer A549 cells to high-LET carbon ion irradiation, which may be due to the inhibition of DNA damage repair and the additional activation of ATM and ATR. Therefore, the results of the present study highlight the potential use of ATM and ATR in clinical radiotherapy for the treatment of NSCLC, since the exposure of lung cancer cells to high-LET rays jointly with NU7026, improved their cellular sensitivity to radiotherapy, compared with irradiation alone. Furthermore, the findings of the present study indicate the use of NU7026 in animal experiments and as a novel agent in gene-radiotherapy, since the compound did not produce any obvious toxic effects on normal lung fibroblasts. Future in vitro and in vivo studies on the combination of DNA-PKcs, ATM and ATR-inhibitors (39) are required in order to assess the beneficial effects of these drugs on the treatment of NSCLC in the clinic.

\section{Acknowledgements}

The authors would like to thank the National Laboratory of Heavy Ion Accelerator, the Gansu Province Tumor Hospital and the Central Laboratory of the School of Life Sciences of Lanzhou University (Lanzhou, China) for their support. The authors would also like to thank the international science editors who provided assistance with the use of the English language during the elaboration of the present manuscript. The present study was supported by the National Natural Science Foundation of China (Beijing, China) (grant no. 81160283).

\section{References}

1. Siegel R, DeSantis C, Virgo K, Stein K, Mariotto A, Smith T, Cooper D, Gansler T, Lerro C, Fedewa S, et al: Cancer treatment and survivorship statistics, 2012. CA Cancer J Clin 62: 220-241, 2012.

2. Jemal A, Siegel R, Xu J and Ward E: Cancer statistics, 2010. CA Cancer J Clin 60: 277-300, 2010.

3. Siegel R, Naishadham D and Jemal A: Cancer statistics, 2012. CA Cancer J Clin 62: 10-29, 2012.

4. Yang HJ, Kim N, Seong KM, Youn H and Youn B: Investigation of radiation-induced transcriptome profile of radioresistant non-small cell lung cancer A549 cells using RNA-seq. PLoS One 8: e59319, 2013.

5. Zhang W, Wang J, Tang M, Pan J, Bai P, Lin D, Qian F, Lin F, Yang $X$ and Zhang S: Quantitative study of lung perfusion SPECT scanning and pulmonary function testing for early radiation-induced lung injury in patients with locally advanced non-small cell lung cancer. Exp Ther Med 3: 631-635, 2012.

6. Hamada N, Imaoka T, Masunaga S, Ogata T, Okayasu R, Takahashi A, Kato TA, Kobayashi Y, Ohnishi T, Ono K, et al: Recent advances in the biology of heavy-ion cancer therapy. J Radiat Res (Tokyo) 51: 365-383, 2010.

7. Janssens GO, Rademakers SE, Terhaard CH, Doornaert PA, Bijl HP, van den Ende P, Chin A, Marres HA, de Bree R, van der Kogel AJ, et al: Accelerated radiotherapy with carbogen and nicotinamide for laryngeal cancer: Results of a phase III randomized trial. J Clin Oncol 30: 1777-1783, 2012.
8. Bussink J, van der Kogel AJ and Kaanders JHAM: Activation of the PI3K/Akt pathway and implications for radioresistance mechanisms in head and neck cancer. Lancet Oncol 9: 288-296, 2008.

9. Nagelkerke A, Bussink J, van der Kogel AJ, Sweep FCGJ and Span PN: The PERK/ATF4/LAMP3-arm of the unfolded protein response affects radioresistance by interfering with the DNA damage response. Radiother Oncol 108: 415-421, 2013.

10. Serra V, Markman B, Scaltriti M, Eichhorn PJ, Valero V, Guzman M, Botero ML, Llonch E, Atzori F, Di Cosimo S, et al: NVP-BEZ235, a dual PI3K/mTOR inhibitor, prevents PI3K signaling and inhibits the growth of cancer cells with activating PI3K mutations. Cancer Res 68: 8022-8030, 2008.

11. Yajima H, Lee K-J, Zhang S, Kobayashi J and Chen BPC: DNA double-strand break formation upon UV-induced replication stress activates ATM and DNA-PKes kinases. J Mol Biol 385: 800-810, 2009.

12. Zhuang W,Li B, Long L, Chen L, Huang Q and Liang Z: Induction of autophagy promotes differentiation of glioma-initiating cells and their radiosensitivity. Int J Cancer 129: 2720-2731, 2011.

13. Stiff T, O'Driscoll M, Rief N, Iwabuchi K, Löbrich M and Jeggo PA: ATM and DNA-PK function redundantly to phosphorylate $\mathrm{H} 2 \mathrm{AX}$ after exposure to ionizing radiation. Cancer Res 64: 2390-2396, 2004.

14. Ghosh S, Narang H, Sarma A and Krishna M: DNA damage response signaling in lung adenocarcinoma A549 cells following $\gamma$ and carbon beam irradiation. Mutat Res 716: 10-19, 2011.

15. Okayasu R, Okada M, Okabe A, et al: Repair of DNA damage induced by accelerated heavy ions in mammalian cells proficient and deficient in the non-homologous end-joining pathway. Radiat Res 165: 59-67, 2006.

16. Vaucher RA, da Motta AS and Brandelli A: Evaluation of the in vitro cytotoxicity of the antimicrobial peptide P34. Cell Biol Int 34: 317-323, 2010.

17. Li Y, Song J, Yang P, Zou R, Fan X and Zhao Z: Establishment of a three-dimensional culture and mechanical loading system for skeletal myoblasts. Cell Biol Int 33: 192-198, 2009.

18. Shen H, Schultz M, Kruh GD and Tew KD: Increased expression of DNA-dependent protein kinase confers resistance to adriamycin. Biochim Biophys Acta 1381: 131-138, 1998.

19. Kantor AF, Li FP, Janov AJ, Tarbell NJ and Sallan SE: Hypertension in long-term survivors of childhood renal cancers. J Clin Oncol 7: 912-915, 1989.

20. Vandersickel V,Depuydt J, Van Bockstaele B, Perletti G, Philippe J, Thierens $\mathrm{H}$ and Vral A: Early increase of radiation-induced $\gamma \mathrm{H} 2 \mathrm{AX}$ foci in a human Ku70/80 knockdown cell line characterized by an enhanced radiosensitivity. J Radiat Res (Tokyo) 51: 633-641, 2010.

21. Yang J, Xu ZP, Huang Y, Hamrick HE, Duerksen-Hughes PJ and Yu YN: ATM and ATR: Sensing DNA damage. World J Gastroenterol 10: 155-160, 2004.

22. Hada M and Georgakilas AG: Formation of clustered DNA damage after high-LET irradiation: A review. J Radiat Res (Tokyo) 49: 203-210, 2008.

23. Wang C and Lees-Miller SP: Detection and repair of ionizing radiation-induced DNA double strand breaks: New developments in nonhomologous end joining. Int J Radiat Oncol Biol Phys 86: 440-449, 2013.

24. Mukherjee B, Kessinger C, Kobayashi J, et al: DNA-PK phosphorylates histone $\mathrm{H} 2 \mathrm{AX}$ during apoptotic DNA fragmentation in mammalian cells. DNA Repair (Amst) 5: 575-590, 2006.

25. Durocher D and Jackson SP: DNA-PK, ATM and ATR as sensors of DNA damage: Variations on a theme? Curr Opin Cell Biol 13: 225-231, 2001.

26. Kim JS, Krasieva TB, Kurumizaka H, et al: Independent and sequential recruitment of NHEJ and HR factors to DNA damage sites in mammalian cells. J Cell Biol 170: 341-347, 2005.

27. Wang H, Wang M, Wang H, Böcker W and Iliakis G: Complex H2AX phosphorylation patterns by multiple kinases including ATM and DNA-PK in human cells exposed to ionizing radiation and treated with kinase inhibitors. J Cell Physiol 202: 492-502, 2005.

28. Yang J, Yu Y, Hamrick HE and Duerksen-Hughes PJ: ATM, ATR and DNA-PK: Initiators of the cellular genotoxic stress responses. Carcinogenesis 24: 1571-1580, 2003.

29. Löbrich M, Shibata A, Beucher A, et al: $\gamma \mathrm{H} 2 \mathrm{AX}$ foci analysis for monitoring DNA double-strand break repair: Strengths, limitations and optimization. Cell Cycle 9: 662-669, 2010.

30. Falck J, Coates J and Jackson SP: Conserved modes of recruitment of ATM, ATR and DNA-PKcs to sites of DNA damage. Nature 434: 605-611, 2005. 
31. Takahashi A and Ohnishi T: Does $\gamma \mathrm{H} 2 \mathrm{AX}$ foci formation depend on the presence of DNA double strand breaks? Cancer Lett 229: 171-179, 2005.

32. Fernandez-Capetillo O, Chen HT, Celeste A, Ward I, Romanienko PJ, Morales JC, Naka K, Xia Z, Camerini-Otero RD, Motoyama N, et al: DNA damage-induced G2-M checkpoint activation by histone H2AX and 53BP1. Nat Cell Biol 4: 993-997, 2002.

33. Stucki M, Stagljar I, Jónsson ZO and Hübscher U: A coordinated interplay: Proteins with multiple functions in DNA replication, DNA repair, cell cycle/checkpoint control, and transcription. Prog Nucleic Acid Res Mol Biol 65: 261-298, 2001.

34. Ferguson DO, Sekiguchi JM, Frank KM, Gao Y, Sharpless NE $\mathrm{Gu}$ Y, Manis J, DePinho RA and Alt FW: The interplay between nonhomologous end-joining and cell cycle checkpoint factors in development, genomic stability, and tumorigenesis. Cold Spring Harb Symp Quant Biol 65: 395-403, 2000.
35. Hong J, Zhang Z, Lv W, Zhang M, Chen C, Yang S, Li S, Zhang L, Han D and Zhang W: Icaritin synergistically enhances the radiosensitivity of 4T1 breast cancer cells. PLoS One 8: e71347, 2013.

36. Yang CJ, Wang CS, Hung JY, Huang HW, Chia YC, Wang PH, Weng $C F$ and Huang MS: Pyrogallol induces $G_{2}-M$ arrest in human lung cancer cells and inhibits tumor growth in an animal model. Lung Cancer 66: 162-168, 2009.

37. Sun Y, Sun D, Li F, Tian L, Li C, Li L, Lin R and Wang S: Downregulation of Sirt1 by antisense oligonucleotides induces apoptosis and enhances radiation sensitization in A549 lung cancer cells. Lung Cancer 58: 21-29, 2007.

38. Zangemeister-Wittke U and Hopkins-Donaldson S: Apoptosis regulation and drug resistance in malignant pleural mesothelioma. Lung Cancer 49 (Suppl 1): S105-S108, 2005.

39. Williams TM, Nyati S, Ross BD and Rehemtulla A: Molecular imaging of the ATM kinase activity. Int J Radiat Oncol Biol Phys 86: 969-977, 2013. 